\title{
Detection of Recurrent Balanced Translocations in Acute Myeloid Leukemia by Multiplex RT-PCR
}

\author{
Jyoti Kotwal ${ }^{1}$ and Manoj Gopal Madakshira ${ }^{2 *}$ \\ 'Dept of Hematology, Gangaram Hospital, New Delhi, India \\ ${ }^{2}$ Dept of Pathology, Armed Forces Medical College, Pune, India
}

\begin{abstract}
Background: Diagnosis of acute myeloid leukemia (AML) has to be established by immunophenotyping and molecular testing for presence of recurrent genetic aberrations. This helps clinician to plan therapeutic protocol as AMLs are a heterogenous group of malignancies having varied response to treatment and prognosis. Multiplex Reverse Transcriptase Polymerase Chain Reaction (RT-PCR) is a method capable of detecting 28 known fusion-transcripts of acute leukemia including cryptic translocations which would otherwise be missed on conventional cytogenetics.
\end{abstract}

Methods: In this case series, a total of 19 consecutive patients diagnosed as AML were included. Morphology and cytochemical character of blasts were ascertained. Immunophenotying was carried out by flowcytometry. Messenager RNA (mRNA) was extracted from peripheral blood or bone marrow aspirates and converted to Complimentary DNA (cDNA). The cDNA was then subjected to multiplex RT-PCR for presence of fusion-transcripts using primers from Hemavision kit, DNA technologies, Denmark. The amplified product was correlated with the morphological, cytochemical and immunophenotypic character of AML.

Result: Of the 19 cases of AML, 12 cases showed amplification of a fusion transcript. Most cases with fusion-transcripts showed an expected blast morphology and immunophenotype. Multiplex RT-PCR picked up presence of rare translocations such as $\mathrm{t}(16,21), \mathrm{t}(9 ; 22), \mathrm{t}(6 ; 9)$ and $\mathrm{t}(3 ; 5)$, with unique clinicopathological features.

Conclusion: Multiplex RT-PCR is a unique assay to identify multiple known recurrent genetic aberrations, including many splice variants. This aids in accurate subtyping of AML and helps the clinician to formulate a specific treatment plan for the patient.

Keywords: Acute Myeloid Leukemia; Multiplex; Polymerase Chain Reaction

\section{Introduction}

The World Health Organisation (WHO) classification of Acute Myeloid Leukemia (AML) mandates the present day diagnostic approach to encompass morphology, cytochemistry, immunophenotying and molecular testing. ${ }^{[1]}$ Each of these modalities of testing has their unique diagnostic relevance and significance. However, there is growing evidence that the disease defining criteria which are prognostically pertinent and therapy oriented are elucidated by the genetic signature of that particular AML subtype. PCR is very much the keystone method of genetic testing as it is a convenient instrument for identification of disease defining recurrent genetic anomalies.

Evaluation of these recurrent genetic aberrations at the molecular level is done by the use of the RT-PCR. This type of PCR begins with the extraction of RNA moieties from the neoplastic cell population. The RNA reflects the transcription of the altered but functional genetic signature. The extracted RNA is converted into cDNA by the help of the reverse transcriptase enzyme. The cDNA is then subjected to a PCR to yield the amplified DNA segment in question. A multiplex PCR has an added advantage wherein multiple DNA segments can be targeted with the help of tailor made primer sequences, thereby identifying the specific genetic aberration amongst many possibilities. Use of multiplex RT-PCR for detection of the various recurrent genetic aberrations in AML has many advantages over the traditional cytogenetics. Of significance include a reduced turn-around time, no mandatory culture of neoplastic cells and a fascinating possibility of detection of cryptic translocations that one may fail to notice while interpreting conventional cytogenetics. ${ }^{[3]}$ Prognostically the detection of these recurrent genetic aberrations yields a sensitive hallmark for subsequent analyses of assessing minimal residual disease following therapy or transplantation. Studies have demonstrated a variable range of up to $25 \%$, in terms of occurrence of false negative cytogenetic analysis when compared to PCR based molecular testing. ${ }^{[4,}$ ${ }^{5]}$ Hence, today PCR based molecular analyses of recurrent genetic aberrations is being increasing used at the time of initial diagnosis of most, if not all AML patients. It is of particular interest to recognise AML patients having 
$\mathrm{t}(8 ; 21), \mathrm{t}(15 ; 17)$ and $\operatorname{inv}(16)$, as these are associated with a relatively favourable prognostication in comparison to the other known genetic aberrations in AML. ${ }^{[1]}$ The endeavor was to study the application of multiplex RT-PCR for detection of presence of recurrent genetic aberrations in cases of acute myeloid leukemia and study their co-relation with clinical presentation.

\section{Materials and Methods}

A total of 19 consecutive cases of AML diagnosed on morphology, cytochemistry and immunophenotyping from July 2010 to August 2012 at a tertiary care centre were included in this prospective pilot study, following clearance from the institutional ethics committee and an informed consent from the patients. The morphological diagnosis was based on peripheral blood smear and bone marrow aspirate. The smears were air-dried and subsequently stained with Leishman-Giemsa stain. Cytochemical stains included Myeloperoxidase (MPO), Periodic Acid Schiff's (PAS) and Non-specific esterase (NSE). The AML was subtyped morphologically as per the French-American-British classification. Bone aspirates or peripheral blood samples were immediately processed at the Flow cytometry laboratory for immunophenotyping of the leukemia. Surface antigens were processed initially followed by cytoplasmic and nuclear antigens. The tagged cell suspension was analysed using the BD FACS Calibur flow cytometer, BD biosciences, USA.

Multiplex-RT PCR was performed by on all samples using the Hemavision kit, DNA technologies, Denmark. This multiplex RT-PCR method is a diagnostic test which is capable of detecting 28 different recurrent genetic aberrations, including more than 80 breakpoints or mRNA splice variants, which have been validated to be specific molecular signatures of acute leukemia subtypes. The first step involved the extraction of RNA from a fresh sample of peripheral blood or bone marrow aspirate using the Qiagen RNA extraction kit, Qiagen, Germany. The extracted RNA was stored at $-80^{\circ} \mathrm{C}$ until the next step. The stored RNA sample was thawed to room temperature and converted to its cDNA using the Verso cDNA kit, Thermoscientific, USA. The cDNA was put through two consecutive nested PCRs with a battery of 8 screening primers designed to locate the segment of interest, if any. Following the nested PCRs the amplified products was run on a $2 \%$ Agarose Gel Electrophoresis. The sample which had any of the 28 possible genetic aberrations, showed up as a positive band in one of the 8 lanes. The sample was subsequently subjected to a second round of split out nested PCRs using primers tailor made to identify the specific genetic aberrations. The amplified product of the second round of split out reactions was run on $2 \%$ Agarose Gel electrophoresis along with a 100 to 1000 base pair molecular base-pair ladder. The recurrent genetic aberration was established by ascertaining the base pair value in relation with the reference ladder on the gel electrophoresis and the key provided in the Hemavision Kit, DNA Technologies, Denmark. An internal control fragment of 911 base pairs was amplified in all reactions, which scrutinizes for the integrity of the extracted RNA and for the probable presence of inhibitors of PCR (Fig 1).

The morphology, cytochemistry and immunophenotype were collated with the multiplex RT-PCR result and a final diagnosis was made as per the 2008 WHO classification of hematolymphoid neoplasms. An excel data sheet was generated to analyse the data using Graphpad Prism statistical software Ver 6.03.

\section{Result}

A total of 19 fresh cases of AML were diagnosed after collating morphology, cytochemistry, immunophenotype and multiplex RT-PCR. Cases of AML which were reported following a relapse were excluded. Adults as expected were the predominant population affected by AML with the median age being 44 years (Table No. 1). AML had a male dominant sex distribution, with 10 of the 19 cases being male patients. 12 cases showed amplification of a fusion transcript, with amplification involving RARA gene, $t(8 ; 21)$ and $t(9 ; 11)$ being the commonest (Graph No. 1). Apart from the common recurrent genetic aberrations mentioned in the $2008 \mathrm{WHO}$ classification, few uncommon translocations, such as $\mathrm{t}(9 ; 22), \mathrm{t}(3 ; 5)$ and $\mathrm{t}(16 ; 21)$ were also amplified (Table No 2). All cases showed an immunophenotype as expected of the WHO classification 2008 subtype (Table No 2). No case of Mixed phenotypic acute leukemia was diagnosed. The mean follow-up period was $6.89+4.581 \mathrm{mths}$ (Range 1-17 months). More than half of the patients (63.15\%) succumbed to the illness during the follow-up period. In terms of response to treatment, 11 cases showed a response to treatment in the form of bone marrow remission, at the end of 1 month. However, one of these 11 cases showed a relapse within the follow-up period (Table No 3).

\section{Discussion}

As per the WHO 2008 classification, any diagnosis of leukemia starts with the morphological examination to document the presence of at least $20 \%$ blasts in bone marrow. ${ }^{[1]}$ The role of cytochemistry is now restricted to identification of MPO, PAS or NSE positive blasts which aid in deciding on the panel of antibodies required for immunophenotyping by flow-cytometry. As per the current WHO guidelines, the primary basis of treatment strategy for AML depends on the immunophenotypic profile of the blasts and presence of any recurrent genetic aberrations. 
Table 1: Demographics, clinical presentation and FAB subtype.

\begin{tabular}{|c|c|c|c|c|c|c|c|c|c|}
\hline Age (yrs) & Sex & Fever & Fatigue & Bleeding & $\begin{array}{c}\text { Hepatol } \\
\text { splenomegaly }\end{array}$ & Adenopathy & $\mathrm{Hb}$ gm/dl & $\begin{array}{l}\text { Platelet / } \\
\text { cumm }\end{array}$ & FAB type \\
\hline 39 & $\mathrm{~F}$ & Present & Present & Absent & Absent & Absent & 10 & 30000 & M0 \\
\hline 62 & $M$ & Present & Present & Present & Absent & Absent & 7 & 110000 & M1 \\
\hline 55 & $\mathrm{~F}$ & Present & Present & Absent & Absent & Absent & 10 & 30000 & M2 \\
\hline 22 & $\mathrm{~F}$ & Present & Absent & Present & Absent & Present & 9 & 13000 & M4 \\
\hline 70 & $\mathrm{~F}$ & Present & Absent & Present & Absent & Absent & 9 & 24000 & M4 \\
\hline 42 & $M$ & Present & Present & Absent & Absent & Absent & 6 & 40000 & M5 \\
\hline 12 days & $\mathrm{M}$ & Present & Absent & Absent & Present & Absent & 8 & 28000 & TAM \\
\hline 45 & $\mathrm{~F}$ & Absent & Present & Present & Absent & Absent & 8 & 35000 & MO \\
\hline 69 & $M$ & Absent & Absent & Present & Absent & Absent & 5 & 50000 & M3 \\
\hline 60 & $M$ & Present & Present & Present & Absent & Absent & 7 & 30000 & M2 \\
\hline 40 & $\mathrm{~F}$ & Absent & Present & Absent & Present & Absent & 8 & 55000 & M2 \\
\hline 76 & $\mathrm{M}$ & Present & Present & Present & Absent & Absent & 7 & 50000 & M 1 \\
\hline 44 & $\mathrm{~F}$ & Present & Present & Absent & Absent & Absent & 10 & 188000 & MO \\
\hline 50 & $M$ & Present & Present & Absent & Present & Absent & 6 & 180000 & M6 \\
\hline 15 & $\mathrm{M}$ & Present & Present & Absent & Absent & Absent & 10 & 90000 & M2 \\
\hline 32 & $M$ & Present & Absent & Absent & Present & Present & 7 & 112000 & M4 \\
\hline 42 & $\mathrm{~F}$ & Present & Present & Present & Absent & Absent & 7 & 120000 & M5 \\
\hline 49 & $\mathrm{M}$ & Present & Present & Present & Present & Absent & 9 & 100000 & M1 \\
\hline 21 & $\mathrm{~F}$ & Absent & Absent & Present & Absent & Absent & 10 & 20000 & M3 \\
\hline
\end{tabular}

Table 2: FAB subtype, immunophenotype, results of MT-PCR and revised WHO subtype

\begin{tabular}{|c|l|l|c|l|}
\hline $\begin{array}{c}\text { FAB } \\
\text { subtype }\end{array}$ & Immunophenotype & MT PCR & $\begin{array}{c}\text { Balanced } \\
\text { translocation }\end{array}$ & Revised WHO classification \\
\hline M0 & CD 34, CD 117, CD13, CD 33 & Positive & $t(16 ; 21)$, & $\begin{array}{l}\text { AML, NOS with minimal } \\
\text { differentiation }\end{array}$ \\
\hline M1 & CD 34, CD 117, CD 33, CD 13 & Positive & $t(9 ; 22)$ & AML, NOS without maturation \\
\hline M2 & CD 33, CD 13, CD 117, MPO, HLA DR & Negative & - & AML, NOS with maturation \\
\hline$M 4$ & $\begin{array}{l}\text { MPO, CD 13, CD 33, HLA DR , CD 117, CD } \\
\text { 34, CD 14, TDT }\end{array}$ & Positive & $\mathrm{t}(6 ; 9)$ & AML with recurrent $t(6 ; 9)$ \\
\hline M4 & CD 33, CD 14, CD 13, CD 117 & Negative & - & AML. NOS myelomonocytic \\
\hline M5 & CD 33, CD 13, CD 14, HLA DR, CD 117 & Positive & inv(16) & AML with recurrent inv(16) \\
\hline TAM & CD117, CD13, CD33, CD7, CD41 & Positive & $t(15 ; 17)$ & AML with recurrent $t(15 ; 17)$ \\
\hline M0 & CD 34, TdT, CD 33, CD 13, CD 117 & Negative & - & $\begin{array}{l}\text { AML, NOS with minimal } \\
\text { differentiation }\end{array}$ \\
\hline
\end{tabular}




\begin{tabular}{|c|c|c|c|c|}
\hline $\begin{array}{l}\text { FAB } \\
\text { subtype }\end{array}$ & Immunophenotype & MT PCR & $\begin{array}{l}\text { Balanced } \\
\text { translocation }\end{array}$ & Revised WHO classification \\
\hline M3 & CD 117, CD 13, CD 33, MPO & Positive & $t(11 ; 17)$ & AML with recurrent $\mathrm{t}(11 ; 17)$ \\
\hline M2 & CD 13, HLA DR, CD 117, CD 34, MPO & Positive & $t(3 ; 5)$ & AML with MDS-related changes \\
\hline M2 & CD 34, HLA DR, MPO, CD 13, CD 19 & Positive & $\mathrm{t}(8 ; 21)$ & AML with recurrent $t(8 ; 21)$ \\
\hline M 1 & CD 33, CD 13, MPO, CD 34, HLA DR & Negative & - & AML, NOS without maturation \\
\hline MO & CD 33, CD 13, CD 117, TdT, HLA DR & Negative & - & $\begin{array}{l}\text { AML, NOS with minimal } \\
\text { differentiation }\end{array}$ \\
\hline M6 & CD 117, Gly A, & Negative & - & AML, NOS erythroid leukemia \\
\hline M2 & $\begin{array}{l}\text { CD 34, HLA DR, MPO, CD 13, CD 15, CD } \\
19\end{array}$ & Positive & $t(8 ; 21)$ & AML with recurrent $t(8 ; 21)$ \\
\hline M4 & $\begin{array}{l}\text { CD 34, CD 13, CD 33, CD 14, HLA DR, CD } \\
117, \text { MPO }\end{array}$ & Positive & $t(9 ; 11)$ & AML with recurrent $t(9 ; 11)$ \\
\hline M5 & CD 13, CD 33, CD 14, HLA DR & Positive & $\mathrm{t}(9 ; 11)$ & AML with recurrent $t(9 ; 11)$ \\
\hline M1 & $\begin{array}{l}\text { CD 117, CD 33, CD 13, MPO, CD 34, HLA } \\
\text { DR }\end{array}$ & Negative & - & AML NOS - Minimal differentiation \\
\hline M3 & CD 33, CD 13, CD 117 & Positive & $\mathrm{t}(15 ; 17)$ & AML with recurrent $\mathrm{t}(15 ; 17)$ \\
\hline
\end{tabular}

Table 3: WHO subtype, response to treatment and outcome.

\begin{tabular}{|c|c|c|c|c|c|}
\hline $\begin{array}{l}\text { Balanced } \\
\text { translocation }\end{array}$ & Revised WHO classification & $\begin{array}{l}\text { Follow-up } \\
\text { Period (mths) }\end{array}$ & $\begin{array}{c}\text { Response to } \\
\text { treatment }\end{array}$ & Outcome & Relapse \\
\hline$t(16,21)$ & $\begin{array}{l}\text { AML, NOS with minimal } \\
\text { differentiation }\end{array}$ & 3 & No & Death & No \\
\hline $\mathrm{t}(9 ; 22)$ & AML, NOS without maturation & 4 & No & Death & No \\
\hline- & AML, NOS with maturation & 7 & No & Death & No \\
\hline $\mathrm{t}(6 ; 9)$ & AML with recurrent $\mathrm{t}(6 ; 9)$ & 4 & No & Death & No \\
\hline- & AML. NOS myelomonocytic & 9 & Yes & Death & No \\
\hline $\operatorname{inv}(16)$ & AML with recurrent inv(16) & 10 & Yes & Death & Yes \\
\hline$t(15 ; 17)$ & AML with recurrent $t(15 ; 17)$ & 1 & No & Death & No \\
\hline- & $\begin{array}{l}\text { AML, NOS with minimal } \\
\text { differentiation }\end{array}$ & 8 & Yes & Death & No \\
\hline$t(11 ; 17)$ & AML with recurrent $t(11 ; 17)$ & 5 & No & Death & No \\
\hline$t(3 ; 5)$ & AML with recurrent $t(3 ; 5)$ & 5 & Yes & Death & No \\
\hline$t(8 ; 21)$ & AML with recurrent $t(8 ; 21)$ & 10 & Yes & Survived & No \\
\hline- & AML, NOS without maturation & 17 & Yes & Survived & No \\
\hline- & $\begin{array}{l}\text { AML, NOS with minimal } \\
\text { differentiation }\end{array}$ & 10 & Yes & Survived & No \\
\hline- & AML, NOS erythroid leukemia & 2 & No & Death & No \\
\hline$t(8 ; 21)$ & AML with recurrent $\mathrm{t}(8 ; 21)$ & 6 & Yes & Survived & No \\
\hline $\mathrm{t}(9 ; 11)$ & AML with recurrent $\mathrm{t}(9 ; 11)$ & 8 & Yes & Survived & No \\
\hline $\mathrm{t}(9 ; 11)$ & AML with recurrent $t(9 ; 11)$ & 2 & No & Death & No \\
\hline- & AML NOS - Minimal differentiation & 17 & Yes & Survived & No \\
\hline$t(15 ; 17)$ & AML with recurrent $t(15 ; 17)$ & 3 & Yes & Survived & No \\
\hline
\end{tabular}




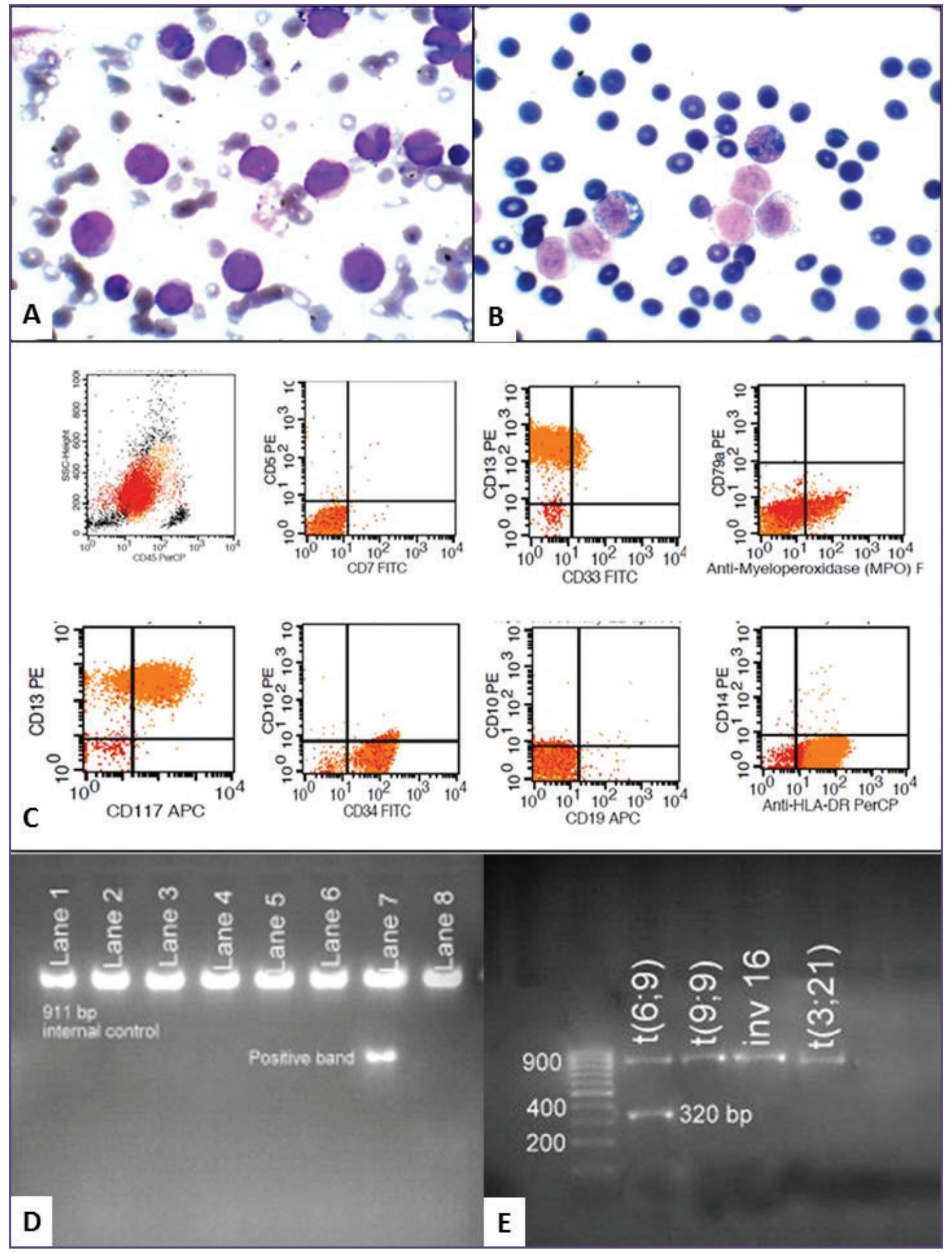

Fig. 1 : Illustration of a case, (A)- Peripheral blood picture with myeloblasts and monoblasts. (B)-MPO positivity in myeloblasts, (C)- Immunophenotype: Positive for CD13, CD33, cMP0 and HLA DR. Negative for CD5, CD7, CD19, CD79a, CD10, CD14, (D)- Agarose gel picture of Master PCR reaction showing positive band in Lane 7, (E)- Agarose gel picture of Spilt out PCR reaction of lane 7 translocations, showing presence of $t(6 ; 9)$ as a 320 bp positive band 


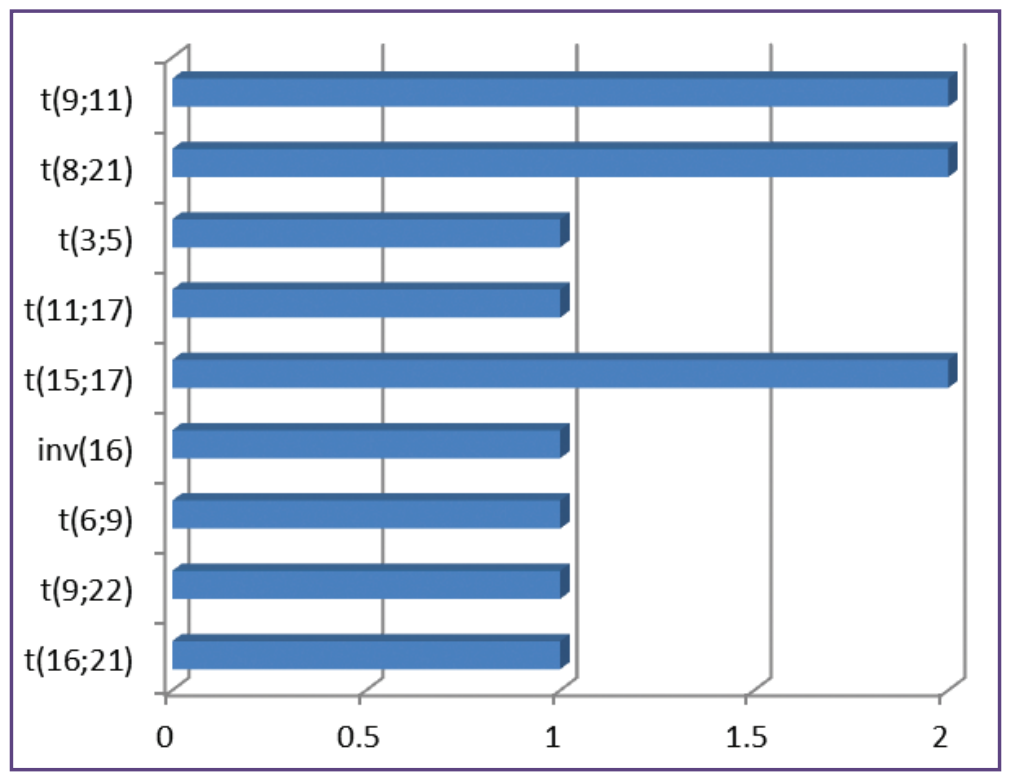

Graph 1: Spectrum of balanced translocations in AML.

[6] This makes it mandatory to subject all leukemic blasts to immunophenotyping and cytogenetic/molecular studies. The utility of molecular assays such as multiplex RTPCR helps to establish presence of any of the known translocations without the need of culture of cells. ${ }^{[2]}$

In our study, a total of 19 AML cases were evaluated. AML cases were seen to affect the adult population nearly $90 \%$ of the cases, with the median age being 44 years. However, literature shows higher peaks of incidence after the sixth decade. ${ }^{[7]}$ This apparent discrepancy can be attributed to the fact that the dependent population of our hospital corresponds to a more young population with higher incidence of de-novo AML rather than those arising from a drug-therapy or myelodysplastic syndromes. There was a slight male preponderance with the male to female ratio of 1.28:1. Various known congenital risk factors have been established to be part of the etiopathogenesis of AML. ${ }^{[8]}$ We had a neonate with Down's syndrome who was found to have Acute Promyelocytic Leukemia (APML) and succumbed to the illness. ${ }^{[9]}$ Of the total AML cases evaluated, 63.15\% (12) of cases showed amplification of a recurrent genetic aberration. The most common amplification was those involving the RARA gene, which is in agreement with the literature. ${ }^{[4,11]}$

Three cases showed amplifications involving the RARA gene, with a wide range of age at presentation, from 12 days, 21 years and 69 years respectively. Though this range of age at presentation can be expected, the most common age at presentation remains adults in their midlife. ${ }^{[11]}$ The neonate in our series presented with fever and hepatosplenomegaly whereas the remaining 02 cases had history of mucosal bleeding and thrombocytopenia. All 03 cases shared a similar and typical immunopheotype of CD13+, CD33+ with absence of HLA-DR. ${ }^{[11]}$ Nevertheless, these cases had slightly different morphology and response patterns. The neonate was initially diagnosed as a case of Transient abnormal myelosis (TAM) which is expected in a case of Trisomy 21 (which was confirmed by conventional karyotyping). However, a postmortem amplification of the archival mRNA demonstrated the presence of a cryptic $t(15 ; 17)$ establishing the diagnosis of APML which was missed on conventional karyotyping done to establish Trisomy 21. ${ }^{[9]}$ The second case was an elderly male (69 years) whose peripheral blood showed cells with large regular nuclei with many eosinophilic granules and occasional Auer rods. A strong MPO positivity was seen in the blasts and the multiplex RT-PCR amplified a variant $\mathrm{t}(11 ; 17)$ translocation. This case did not respond to therapy with ATRA and succumbed to the illness. It has been documented that patients with variant $t(11 ; 17)$ do not respond well to conventional ATRA therapy. ${ }^{[12]}$ The third case presented with a deceptively low leucocyte count with few atypical hypogranular promyelocytes seen in the tail of the peripheral smear. The suspicion of variant APML was confirmed by demonstration of the translocation $\mathrm{t}(15 ; 17)$. The patient responded well to therapy with ATRA and was in hematological remission during the last follow up period. ${ }^{[13]}$ The association of FLT3-ITD mutations has been seen in $35-45 \%$ of APML cases, which would push them into the intermediate risk category. ${ }^{[14]}$ Though unavailable at our center, all cases of APML should be screened for this mutation to better stratify the disease for better therapeutic management. 
We encountered 02 cases with $t(8 ; 21)$ which were seen in a 15 year boy and a 40 year lady. Both patients had characteristic myeloblasts with perinuclear clearing accompanied by a significant proportion of cells with maturation, fitting into the FAB category of M2. No evidence of tumor manifestations in the form of myeloid sarcoma, expected in such subtypes, was seen in our cases. Blasts in both cases demonstrated an immunophenotype of $\mathrm{CD} 13+, \mathrm{MPO}+$ and HLA-DR+. Blasts in one of the case expressed the lymphoid marker CD19, which has been reported in such subtypes. ${ }^{11}$ Both cases showed a good response to chemotherapy and were in complete hematological remission during the last follow-up period. It would be of value if such cases are screened for possible KIT mutation, which would require a more aggressive management to maintain remission status. ${ }^{[15]}$

Two patients, who were in their 3rd and 4th decade of life, were detected to have amplification of $t(9 ; 11)$, though this subtype is more commonly encountered in children. One of the patients had a predominant monocytic component deserving a FAB subtype of M5. Whereas the other patient showed a significant subgroup of neutrophilic precursors, thus prompting a label of M4 FAB subtype ${ }^{[11]}$ The monocytic component known to be associated with this translocation showed a strong NSE reaction in both the cases. The immunophenotype confirmed the monocytic component, with blasts being positive for CD14. The response to therapy was contrasting in the two cases; with the younger patient going into remission while the other patient, having a higher blast count succumbed to the illness. ${ }^{[16]}$

A 39 year old lady presented with history of fever and fatigue, with her peripheral blood showing myeloblasts with minimal differentiation and MPO being positive in $<3 \%$ of blasts. The multiplex RT-PCR amplification showed presence of translocation $\mathrm{t}(16 ; 21)$ which is seen only in rare cases of de-novo AML, but is commonly associated with Ewing's sarcoma. Morphology of such cases usually show myelocytic and/or monocytic features; with few cases showing a prominent eosinophilic component, which was absent in our case. ${ }^{[17]}$ The blasts in this case has a CD34+, C117+, CD13+ and CD33+ immunophenotype. Cases carrying this translocation have a dismal prognosis, as seen in our patient who succumbed to the disease within 3 months of diagnosis. ${ }^{[17]}$

We had an interesting case of a 62 year man who presented with fever, fatigue and mucosal bleeding. His peripheral blood had myeloblasts with absence of maturation, fitting into a FAB subtype of M1. The mRNA extracted from the blasts showed an amplification of $t(9 ; 22)$ on multiplex RTPCR. Such subtypes can have any FAB morphology other than $\mathrm{M} 3$ and $\mathrm{M} 7$, and usually present with splenomegaly in $50 \%$ of cases. ${ }^{[18]}$ The immunophenotype was on expected lines with positivity for CD34, CD117, CD33 and CD13. These patients are known to have a poor outcome, and the response is not significantly different even after addition of tyrosine kinase inhibitors. Hence, AML with $t(9 ; 22)$ has been recommended to be placed in the group of poor cytogenetic risk factors. ${ }^{[18]}$ Our patient succumbed to the illness within 4 months of diagnosis, though tyrosine kinase inhibitors were not part of the therapy.

In this series, we demonstrated amplification of $t(6 ; 9)$ in a 22 year old lady, who presented with fever, mucosal bleeding and significant cervical lymphadenopathy. The peripheral blood had cells of mixed myelomonocytic lineage. No significant basophils were seen, both in the peripheral blood and bone marrow aspiration smears, which has been reported in most cases of this subtype. ${ }^{[19]}$ Neither, was any dyspoietic changes detected in the bone marrow aspirates. The blasts showed a non-specific myeloid immunophenotype with positivity for CD13, CD33 and HLA-DR. The patient did not go into remission and succumbed to the illness, re-asserting the poor prognosis of this translocation. ${ }^{[19]}$

Asingle case withinv(16) amplification was seen. The patient was a 42 year old male with presenting complaints of fever and fatigue. The bone marrow showed a slight prominence of eosinophilic precursors which is characteristically seen in such leukemic subtypes. ${ }^{[2]}$ The blasts had an expected immunophenotype with positivity for CD13, CD33, HLADR, CD117 and CD14. Though patients with inv(16) are known to have complete remissions, our patient succumbed to the disease after achieving an initial period of remission. This may have been due to the presence of KIT mutations, seen in approximately $30 \%$ of patients, which confers a worse survival outcome. ${ }^{15}$ However; analysis for this mutation was not done in this case.

Another rare translocation $(3 ; 5)$ was amplified involving the NPM1 gene. This translocation is usually seen in elderly patients who develop AML with a prior history of myelodysplasia. ${ }^{[2]]}$ Our patient was an elderly male who was being treated as a case of Refractory anemia with excess blasts (RAEB-2). At the time of diagnosis, the mandatory $20 \%$ blasts were seen with concurrent evidence of dyspoietic changes in both granulocytic and erythroid series. The blasts carried the immunophenotype of $\mathrm{CD} 34+, \mathrm{CD} 13+, \mathrm{CD} 33+, \mathrm{MPO}+$ and CD117+. The patient had a rapid down-hill course with worsening transfusion dependent anemia and persistent fever in spite of therapy and had a fatal outcome. This is in concordance with the literature which predicts a poor prognostic outcome in such cases. ${ }^{[21]}$ 
Molecular diagnosis of AML has become mandatory with the advent of the WHO 2008 classification. Amongst the various diagnostic modalities, use of multiplex RT-PCR for identification of known genetic aberrations has many advantages. ${ }^{[3]}$ However, there is a need for critical judgment and insight for prediction of disease outcome, progress and response, as optimal therapeutic decisions will be based on accurate classification of the AML subtype at the time of initial diagnosis. Detection of specific fusion-transcripts helped in accurate sub-typing of AML, benefiting patients by guiding specific management protocols and follow-up regimens. Most cases with amplified genetic aberrations had a poor prognosis with no significant difference seen in comparison to cases which no amplification on multiplex RT-PCR. However, few cases of AML with core-binding factors showed a better response to therapy. The multiplex RT-PCR including the split-out phase reactions were completed within 2 days and the assay was amenable to analysis of up to 10 samples simultaneously.

This study demonstrates the utility of multiplex RT-PCR as a clinically useful, efficient and fast procedure for detection of genetic changes in AML. Multiplex RT-PCR has various advantages, but is also fraught with disadvantages. The entire assay involves 6 steps of amplification and many variables in the form of adequacy of blasts, maintenance of cold chain and viability of primers and reagents. However, the presence of the in-built control in each reaction confirmed whether the downstream steps were performed meticulously. ${ }^{[22]}$ Nevertheless, multiplex RTPCR is unable to detect certain chromosomal anomalies such as hyperdiploidy, hypoploidy and deletions, which can only be discerned by conventional cytogenetics. The inclusion of cytogenetic analysis would have acted as a good comparative assay for correlating our findings and arrive at a more decisive opinion.

\section{Conclusion}

We believe that the MT-PCR assay is clinically useful as an efficient and rapid assay for the detection of genetic aberrations in AML that would complement cytogenetics in a fruitful manner. Multiplex RT-PCR is a unique approach to address the new discoveries of genetic aberrations in AML as it is open for addition of new primer sets as information of new aberrations are validated. This assay can be extended for detection of minimal residual disease in AML, because the split-out reaction has a sensitivity equivalent to that of single PCR assays. This makes the multiplex RT-PCR approach significant not only in establishing the diagnosis, but also for subsequent clinical decision-making.

\section{Acknowledgements :}

Dr Rajan Kapoor, Clinical Hematologist, Army Hospital (Research \& Referral), New Delhi

\section{Reference}

1. Vardiman JW, Brunning RD, Arber DA et al. Introduction and overview of classification of the myeloid neoplasms. In: Swerdlow SH, Campo E, Harris NL, Jaffe ES, Pileri SA, Stein $\mathrm{H}$ et al, editors. WHO Classification of Tumors of Haematopoietic and Lymphoid Tissues. Lyon, France: IARC Press, 2008; p. 18-30

2. Olesen LH, Clausen N, Dimitrijevic A, Kerndrup G, Kjeldsen E, Hokland P. Prospective application of a multiplex reverse transcription-polymerase chain reaction assay for the detection of balanced translocations in leukaemia: a singlelaboratory study of 390 paediatric and adult patients. Br J Haematol. 2004; 127(1):59-66

3. Hutchings HM, Wirenfeldt KT, Hasle H, Schmiegelow $\mathrm{K}$, Brondum-Nielsen K, Johnsen HE. Multiplex reverse transcriptase polymerase chain reaction screening in acute myeloid leukemia detects cytogenetically unrevealed abnormalities of prognostic significance. Haematologica. 2005; 90(7):984-986

4. Salto-Tellez M, Shelat SG, Benoit B et al. Multiplex RTPCR for the detection of leukemia-associated translocations: validation and application to routine molecular diagnostic practice. J Mol Diagn. 2003; 5(4):231-236

5. Strehl S, König M, Mann G, Haas OA. Multiplex reverse transcriptase-polymerase chain reaction screening in childhood acute myeloblastic leukemia. Blood. 2001 ;97(3):805-808

6. Khalidi HS, Medeiros LJ, Chang KL, Brynes RK, Slovak ML, Arber DA. The immunophenotype of adult acute myeloid leukaemia: high frequency of lymphoid antigen expression and comparison of immunophenotype, FAB classification, and karyotypic abnormalities. Am J Clin Pathol 1998; 109(2):211-220

7. Head DR. Diagnosis and classification of the acute leukemia and myelodysplastic syndromes. In: Greer JP, Foerster J, Rodgers GM, Paraskevas F, Glader B, Arber DA et al editors. Wintrobe's clinical hematology. 12th ed: Lippincott Williams; 2009.p.1809-1819

8. Sandler DP, Collman GW. Cytogenetic and environmental factors in the etiology of the acute leukaemias in adults. Am J Epidemiol 1987; 126:1017-1032

9. Kotwal J, Manoj MG, Malik A, Sinha K, Singh D, Dutta V. Role of multiplex reverse-transcriptase PCR for diagnosis of Acute myelosis in an infant with Down's syndrome. Med J Armed Forces India. 2015;71:S12-5

10. Choi HJ, Kim HR, Shin MG, Kook H, Kim HJ, Shin JH, et al. Spectra of Chromosomal Aberrations in 325 Leukemia Patients and Implications for the Development of New Molecular Detection Systems. J Korean Med Sci. 2011; 26(7):886-92

eISSN: 2349-6983; pISSN: 2394-6466 
11. Arber DA, Brunning RD, Le Beau MM, Falini B. Acute myeloid leukemia with recurrent genetic abnormalities In: Swerdlow SH, Campo E, Harris NL, Jaffe ES, Pileri SA, Stein H, Thiele J, Vardiman JW (Eds): WHO classification of Tumors of haematopoietic and Lymphoid Tissues. IARC: Lyon; 2008.p.110-123

12. Licht JD, Chomienne C, Goy A, Chen A, Scott AA, Head $\mathrm{DR}$, et al. Clinical and molecular characterization of a rare syndrome of acute promyelocytic leukemia associated with translocation (11;17). Blood 1995; 85:1083

13. Guidez F, Ivins S, Zhu J, Söderström M, Waxman S, Zelent A. Reduced retinoic acid-sensitivities of nuclear receptor co-repressor binding to PML-and PLZF-RAR $\alpha$ underlie molecular pathogenesis and treatment of acute promyelocytic leukemia. Blood.1998;91(8):2634-2642

14. Gilliland DG, Griffin JD. The roles of FLT3 in hematopoiesis and leukemia. Blood 2002;100:1532-1542

15. Paschka P, Marcucci G, Ruppert AS, Mrozek K, Chen H, Kittles R A et al (2006). Adverse prognostic significance of KIT mutations in adult myeloid leukemia with inv(16) and $\mathrm{t}(8 ; 21)$ : a Cancer and Leukemia Group B study. J Clin Oncol 24:3904-3911

16. Mrózek K, Heinonen K, Lawrence D et al. Adult patients with de novo acute myeloid leukemia and $\mathrm{t}(9 ; 11)(\mathrm{p} 22 ; \mathrm{q} 23)$ have a superior outcome to patients with other translocations involving band 11q23: a cancer and leukemia group B study. Blood. 1997;90(11):4532-8

17. Sadamori N, Yao E, Tagawa $M$ et al. 16;21 translocation in acute non lymphocytic leukemia with abnormal eosinophils: a unique subtype. Acta Haematol. 1990; 84(4):212-6

18. Gopal MM, Kotwal J, Kapoor R. Acute Myeloid Leukemia with BCR/ABL Fusion Chimera. Indian Journal of Hematology and Blood Transfusion. 2014;30(1):280-2

19. Oyarzo MP, Lin P, Glassman A, Bueso-Ramos CE, Luthra R, Medeiros LJ. Acute myeloid leukemia with t (6; 9)(p23; q34) is associated with dysplasia and a high frequency of flt3 gene mutations. American journal of clinical pathology. 2004;122(3):348-58

20. Delaunay J, Vey N, Leblanc $\mathrm{T}$ et al. Prognosis of inv $(16) / t(16 ; 16)$ acute myeloid leukemia (AML): a survey of 110 cases from the French AML Intergroup. Blood. 2003;102(2):462-9

21. Raimondi SC, Dubé ID, Valentine MB et al. Clinicopathologic manifestations and breakpoints of the $t(3 ; 5)$ in patients with acute non lymphocytic leukemia. Leukemia. 1989; 3(1):42-7

22. Song MJ, Kim HJ, Park $\mathrm{CH}$ et al. Diagnostic utility of a multiplex RT-PCR assay in detecting fusion transcripts from recurrent genetic abnormalities of acute leukemia by WHO 2008 classification. Diagnostic molecular pathology. 2012;21(1):40-4

*Corresponding author:

Dr Manoj Gopal Madakshira, Dept of Pathology, Armed Forces Medical College, Pune-411040, INDIA

Phone: +91 8551926889

Email: manoj.gopal@gmail.com

Date of Submission : 13.09.2016

Date of Acceptance : 24.12.2016

Financial or other Competing Interests: None.
Date of Publication : 23.02.2017 